\title{
Die Pflanzenverwendung von Gustav Allinger
}

\author{
Swantje Duthweiler
}

$\mathrm{P}$ flanzenverwendung ist ein Aspekt in der Gartenkunst, der durch die Kurzlebigkeit wesentlicher Elemente, wechselnder Pflegeintensitäten und neuer Bepflanzungsmoden nicht immer in seiner authentischen Wirkung überliefert ist. Von vielen Pflanzkonzepten und ausgeführten Pflanzungen früherer Generationen von Gartenarchitekten gibt es kaum oder keine Dokumentation. Anders ist es bei Gustav Allinger (1891-1974), der zu Lebzeiten immer wieder über seine Pflanzideale im Zusammenhang mit seiner Gesamtplanung berichtete und diese auch mit Fotos und manchen Plänen dokumentierte. Im Folgenden soll nachvollzogen werden, ob und wie sich die Pflanzenverwendung von Gustav Allinger innerhalb seiner Hauptwirkungszeit zwischen 1929 und 1941 verändert hat.

\section{Sozialreformerische Ideale der Reform- gartenzeit}

Eine der ersten überregional bekannten Parkgestaltungen und Pflanzungen von Gustav Allinger war die Jubiläums-Gartenbauausstellung in Dresden 1926 - die erste große Gartenbauausstellung in Deutschland nach dem Ersten Weltkrieg. Allinger hatte als junger Berliner Gartenarchitekt für die Planungsabteilung der Baumschule Späth den Entwurfswettbewerb zur Gesamtgestaltung gewonnen und erhielt daraufhin die künstlerische Leitung. In wenigen Jahren schuf er im Großen Garten in Dresden auf 30 Hektar das größte und umfassendste farbliche Gesamtkunstwerk der Moderne. Konzeptionell standen Allingers Pflanzun- gen damals noch eng in der Tradition der Teppichbeete, wirkten in ihrer monumentalen Weite aber eher als Farbfelder, als Beete. Er plante seine Pflanzungen mit einer ganz neuartig emotionalen Radikalität und betonte 1926 den Wunsch, die »weiten Tulpenund Narzissenblütenfelder (Hollands) vor den Augen jener armen Menschen ausschütten zu dürfen, die nie in ihrem Leben das Glück hatten, solche Blumenherrlichkeit zu schauen ${ }^{1}{ }^{1}$

Als ehemaliger Lehrling und späterer Mitarbeiter Fritz Enckes (1861-1931) war Allinger deutlich von dessen sozialreformerischen Leitideen beeinflusst, eine Haltung, die man auch beim Encke Schüler Erwin Barth (1880-1933) findet. Doch während Encke und Barth als Ersatz für fehlende Hausgärten in dicht besiedelten Stadtteilen staudenreiche Rabatten und übersichtliche Teppichbeete pflanzten, entschied sich Allinger für eine deutlich größere Geste und lautere Wirkung. Er entwickelte bei seiner Farbgestaltung der Jubiläums-Gartenbauausstellung in Dresden nicht nur den Gesamtentwurf der Gartenschau, sondern bearbeitete nahezu die Hälfte der 44 Einzelprojekte selbst ${ }^{2}$ - zeitgenössischen Berichten zufolge hatten sich »Komitee und Aussteller ... untergeordnet $«{ }^{3}$ Allinger entwarf für den Großen Garten in Dresden zwei große Farbachsen - den »Riesengroßen Rosengarten ", in großen Blöcken abgetönt von Dunkelrot über Rosa- und Lachsfarben bis Orange, Gelb und Weiß und als Kontrapunkt dazu den »Garten zum blauen Rittersporn«(Abb. 1). Für den »Garten zum blauen Rittersporn« beauftragte Allinger 
Karl Foerster (1874-1970) mit einer Delphinium-Massenpflanzung, die dieser mit niedrigen, buschigen, standfesten Sorten erfüllte. Den Rittersporngarten verlängerte er mit violetten Farbenfeldern der Einjahresblumen (Abb. 2) und ergänzte die Gesamtgestaltung systematisch mit den Farbenfeldern gärtnerischer Sondergärten. Insgesamt muss die zeitgenössische Reaktion überwältigend gewesen sein. Camillo Schneider berichtete von einer »Sehenswürdigkeit ersten Ranges« und in der Massenwirkung "einzig schön «. ${ }^{4}$ Schneider stellte fest, dass der große Erfolg beim Publikum gezeigt hätte, wie »blumenhungrig $~^{5}$ die (Menschen-)Massen gewesen seien.

\section{Vom Teppichbeet zum Farbfeld}

Hintergrund dieser Pflanzweise waren zeitgenössische Diskussionen über Teppichbeete und potentielle Weiterentwicklungsmöglichkeiten. 1912 befragte die einflussreiche Fachzeitschrift »Möller's Deutsche Gärtner-Zeitung « führende Gartenarchitekten und Stadtgartendirektoren nach ihrer Meinung zum Teppichbeet und seiner modernen Weiterentwicklung. Bei dieser Umfrage hatten sich überraschend viele Kollegen für eine »künstlerische Wiedergeburt« des Teppichbeetes ausgesprochen. ${ }^{6} 1918$ forderte der Dresdner Gartenarchitekt Hans Felix Kammeyer (1893-1973) sogar, von der reichhaltigen Staudenrabatte "wieder abzukommen«. Die »Blume als Massenwirkung« müsse die Gärten erobern:

»Unser Voreltern wussten schon was sie taten, als sie das Teppichbeet schufen! «7

Die Zeit des Expressionismus war deutlich in den Beeten zu erleben, farbintensiv und sehr emotional. Schon 1913 hatte Leberecht Migge (1881-1935) auf der Internationalen Baufachausstellung in Leipzig in dieser Tradition gearbeitet und lange Gartenachsen mit leuchtend bunten Teppichbeetpflanzungen kombiniert. Migge sah seine Leipziger
Pflanzungen als Erneuerung des "viel befeindeten Gartenmotivs des Teppichbeetes ${ }^{8}{ }^{8}$ In diesem Zusammenhang kann man Allingers Pflanzweise in Dresden als überproportional großes dekoratives Teppichbeet verstehen, das nicht nur aus Sommerblumen, sondern auch aus Stauden und Rosen zusammengesetzt wurde. Um die für Teppichbeete charakteristische Aufsicht auf die umfangreiche Pflanzung zu gewährleisten, wurde ein Turm gebaut - der sogenannte »Grüne Dom«. Größere Gehölze waren innerhalb der Achsen nicht vorgesehen, um den Blick auf den Pflanzenteppich nicht zu behindern.

Nach seinem großen Erfolg 1926 in Dresden wiederholte Allinger 1927 die farbintensiven Massenpflanzungen auf der Deutschen Gartenbau- und Schlesischen Gewerbeausstellung in Liegnitz. Doch zu Beginn der 1930er Jahre ist in Deutschland eine allgemeine Stiländerung zu beobachten. Spätestens ab der Weltwirtschaftskrise 1930 spielten expressionistische Sommerblumenfelder in der Gartenarchitektur keine Rolle mehr. In den Umbruchszeiten findet man immer wieder sehr kritische Stimmungen zur expressionistischen Gartenkunst, die angesichts allgemein wirtschaftlicher Not nicht mehr für angebracht galten. Gewächshäuser konnten nicht mehr zur Sommerblumenanzucht reserviert und geheizt werden, stand in den Notzeiten die Blumenproduktion in direkter Konkurrenz zur lebensnotwendigen Gemüse- und Obstproduktion. Expressionistisch planende Gartenarchitekten hätten »interessens- und verständnislos allem persönlichen Erleben der Liebhaber gegenüber « gearbeitet und damit »wesenlose Gärten « geschaffen, vor denen der Besitzer »entmutigt « stehe und vergeblich nach einem inneren Anschluss suche $«{ }^{9}$ Auch Karl Foerster kritisierte die "großflächige Farbenmonotonie« als kalte Pracht und verglich die »allgemein phantasielose Pflasterung « von Beeten mit einer Sorte mit dem »Liebeslied eines Mädchens gesungen von einem »Männergesangverein «. ${ }^{10}$ 


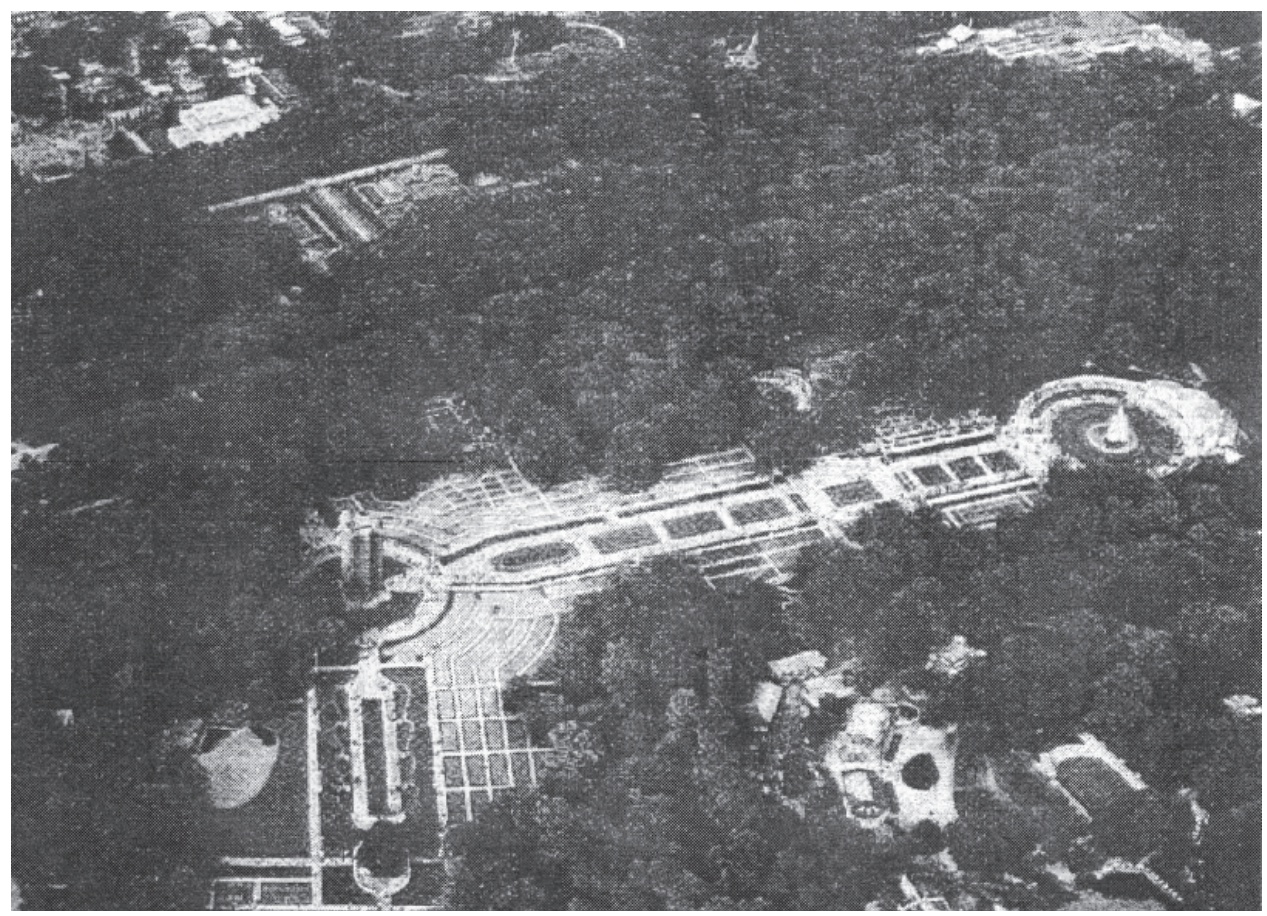

1 | Jubiläums-Gartenbauausstellung in Dresden 1926 (Gesamtentwurf und Pflanzplanung: Gustav Allinger), Fotografie 1926 (Gustav Allinger: Die Jubiläums-Gartenbau-Ausstellung Dresden, Berlin 1926, S. 4).

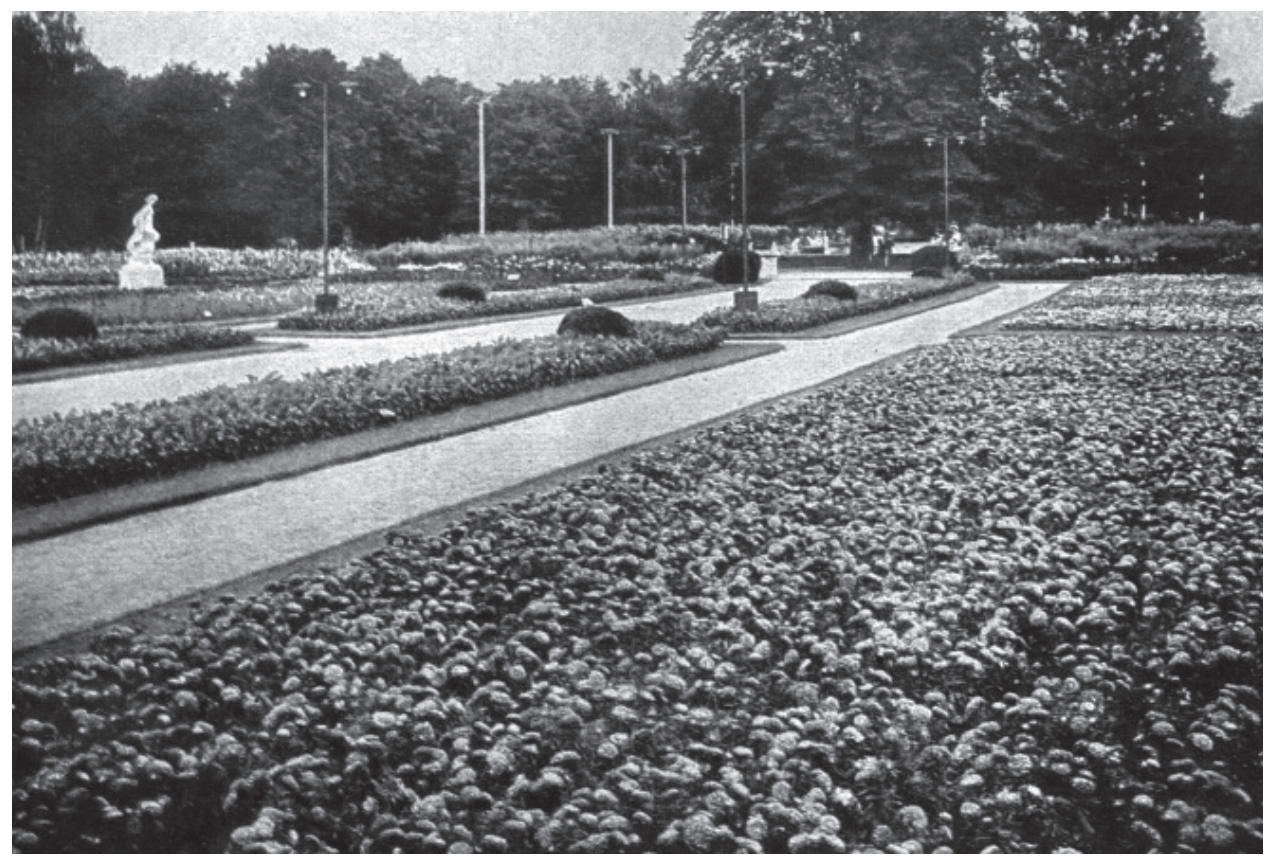

2 | Farbenfelder der Einjahresblumen auf der Jubiläums-Gartenbauausstellung in Dresden 1926 (Die Gartenwelt, 1927, S. 182). 


\section{Die Wiederentdeckung der Staude}

1936 betonte Allinger rückblickend, er hätte nach Jahren, in denen er pflanzliches Material ganz der Form des Gartens untergeordnet hätte, sich mehr den Pflanzen selbst zugewandt. ${ }^{11}$ In seinen Texten nennt Allinger als neues Leitbild Landschaftselemente mit entsprechend "physiognomisch dazu stimmenden Pflanzen « - die gestalterische Überhöhung eines Landschaftsbildes mit einheimischen und fremden Pflanzen im Sinne von Willy Lange (1864-1941). Nicht nur Willy Lange und Karl Foerster hatten hier anscheinend Einfluss auf den Berliner Gartenarchitekten Allinger gehabt. Schon sein Mentor Fritz Encke war einer der ersten in Deutschland, die in öffentlichen Gartenanlagen eine üppige und bunte Blütenstrauch-, Stauden- und Sommerblumenfülle im Kontrast zu streng architektonischer Linienführung setzte (Abb. 3). Allinger schrieb 1936 über Encke: »Wer je Einblick in das Wesen und das Werk dieses bedeutenden Gartenkünstlers [Fritz Encke] gewinnen durfte, weiß auch, dass es nie einen Gartengestalter gegeben hat, dessen Herz der Pflanze und der Natur inniger zugetan war «. ${ }^{12}$ Encke zufolge waren Pflanzen "naturgemäß der Hauptinhalt des Gartens «. ${ }^{13}$

In seinen Schriften beschrieb Allinger unter anderem ausführlich die Gestaltung des Hermann-Löns-Parks in Hannover durch Wilhelm Hübotter (1895-1976) als künstliche Auenlandschaft mit weiten Wiesenflächen, für den man vor Beginn der Arbeiten ein pflanzensoziologisches Gutachten von Reinhold Tüxen (1899-1980) beauftragt habe. ${ }^{14}$ Eine "landschaftliche Pflanzweise unter Betonung heimatlicher Pflanzengesellschaften « lernte Allinger schon bei Fritz Encke kennen, der in dieser Weise 1905 den Klettenbergpark in Köln bearbeitet hatte. ${ }^{15}$ Das Pflanzkonzept sei dabei ganz auf den individuellen Ort abzustimmen, »in vollkommener Harmonie mit dem Genius Loci«. ${ }^{16}$

In dieser Phase folgten Allingers nächsten
Parkplanungen und Pflanzungen, wie zum Beispiel der Sport- und Waldpark eines Werkes in Dessau - in Gemeinschaftsarbeit mit dem Berliner Architekten Werner Issel (1884-1974) - der Richard-Wagner-Park in Bayreuth (ab 1929) und der Richard-Wagner-Hain in der ehemaligen Elster-PleißeAue in Leipzig. Auch für seinen eigenen Parkanlagen der 1930er Jahre betonte Allinger 1941 den pflanzensoziologischen Hintergrund der waldartigen Rahmenbepflanzung der »Haine «. ${ }^{17}$ Vergleicht man diese einfühlsamen Theoriem mit der Pflanzpraxis im Richard-Wagner-Hain in Leipzig ist man erstaunt. Eine massive Quadratraster-Pflanzung aus Linden-Hochstämmen bildet hier klare Raumkanten und eine ruhige Rahmung von Sondergärten als hausgartenähnliche Rückzugsräume und des eigentlichen Denkmalsplatzes. Der bereits 1932 zur Planung beauftragte RichardWagner-Hain wurde bei seiner Grundsteinlegung 1934 nachträglich nationalsozialistisch als »Richard-Wagner-National-Denkmal« ideologisiert. Ursprünglich war zum Gedenken an den 50. Todestag des in Leipzig geborenen Richard Wagner nur ein Einzeldenkmal auf einem Platz vorgesehen. Allinger berichtet, dass ihm dieses Projekt als zu unbedeutend erschien und er den Oberbürgermeister und den Stadtbaurat stattdessen vom Bau einer umfangreichen Parkanlage überzeugen konnte. Als Wagner-Ehrung sollten Natur und Kunst zu einer »einmaligen Landschaft und würdigen Weihestätte« verschmelzen. ${ }^{18}$ Die Gesamtanlage sollte in »Größe der Auffassung, in der Schönheit der Landschaft und ... als schöpferische Einheit sich des großen Meisters [Richard Wagner] ... würdig erweisen $« .{ }^{19}$ Weite Staudenflächen, Rasenlandschaften, Gehölzrasterpflanzungen und ein Lindengang als Aussichtspromenade - der Richard-WagnerPark in Bayreuth beziehungsweise der Richard-Wagner-Hain in der ehemaligen Elster-Pleißen-Aue in Leipzig (Abb. 4) entsprachen einem Gesamtgedanken, dem sich alle Sondergärten und alle Pflanzungen unterzuordnen hatten. Im Richard-Wagner- 


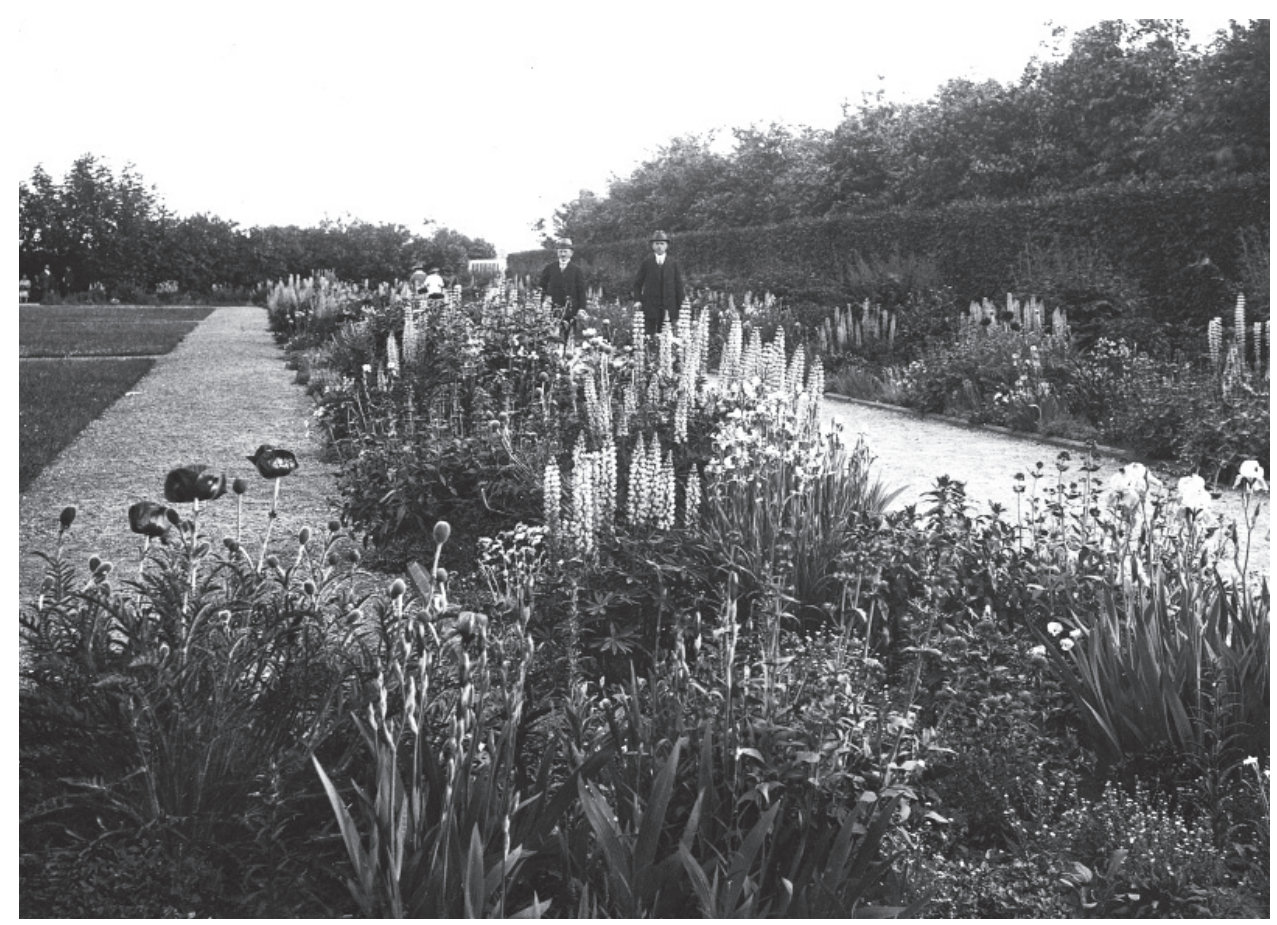

3 | Staudenrabatte von Fritz Encke im Vorgebirgspark in Köln, Planung 1911-14, Fotografie, Richard Stoll um 1920 (Archiv Amt für Landschaftspflege und Grünflächen Köln, Glaspositiv ohne Archivnummer).

Hain in Leipzig hatte Allinger einen architektonischen Volkspark gebaut mit einer »Verwendung der Pflanzen und Blumen im Sinne einer malerischen Belebung des Gartens . $^{20}$

\section{Von der ländlichen Rabatte zur monumentalen Pflanzung}

So ist zu beobachten, dass Allinger heimische Landschaftsbilder eher als gestalterisches Leitbild ansieht, denn als naturwissenschaftliche Aufgabe. Schon beim Sport- und Waldpark in Dessau hätte er den vorhandenen Gehölzbestand mit »Kiefern, Rotbuche, Weißbuchen, Birken, Akazien" einbezogen und die weitere Pflanzung »im Grundsätzlichen auf eine pflanzensoziologische Einheit abgestimmt«. Doch fügt der nationalsozialistisch überzeugte Planer einschränkend hinzu: »soweit es sich um den großen Rahmen der Anlagen handelt $« .{ }^{21}$ An verschiede- nen Stellen (Sondergärten) sei es durchaus erwünscht und notwendig, "ausgesprochene Garten- und Blütenpflanzen bei der Gestaltung zu verwenden ${ }^{22}$. Bei der Gestaltung des Richard-Wagner-Hains findet man anhand der überlieferten Pflanzpläne zwischen heimischen Eichen (Quercus robur) und Ahornarten auch amerikanische Rot-Eichen mit besonderer Herbstfärbung (Quercus rubra), blühintensive Kastanien (Aesculus hippocastanum) und formdominante Pyramiden-Pappeln (Populus nigra 'Italica'). Feingefiederte heimische Sand-Birken (Betula pendula) wurden ergänzt durch amerikanische Scheinakazien (Robinia pseudoacacia) und chinesische Götterbäume (Alianthus altissima). So ist festzustellen, dass Allingers Pflanzcharakter insgesamt eher dekorativ als naturnah wirkte und in diesem Ausdruck durch farbintensive Blütensträucher, Kletterpflanzen und Stauden weiter vertieft wurde. 


\section{Die Pflanzenverwendung von Gustav Allinger}

Bei den Sträuchern findet man im RichardWagner-Hain immer wieder Kontraste von dunklen, oft immergrünen Gehölzen zu farbintensiven, freiwachsenden Arten. Pyramiden-Pappeln und Robinien wurden von Wällen aus Rhododendron und Forsythie eingefasst, immergrüne Gehölzschirme aus Eibe (Taxus baccata), Pfitzers Wacholder (Juniperus x pfitzeriana), Zwerg-Kriechwacholder (Juniperus sabina 'Tamariscifolia') oder Feuerdorn (Pyracantha coccinea) bildeten den Hintergrund für duftende, rosablühende Borstige Robinien (Robinia hispida) und Japanische Azaleen (Rhododendron molle). Selbst die Kletterpflanzen an den
Säulenhallen nahmen dieses Thema auf, kombinierte Allinger Schling-Knöterich (Fallopia baldschuanica, syn. Polygonum aubertii) mit Wildem Wein (Parthenocissus quinquefolia var. engelmannii) und leuchtend roten Schling-Rosen (Rosa 'Paul's Scarlet Climber').

Im eigenen Lehrbuch »Der deutsche Garten « betont Allinger: »Wenn auch die Form eines Gartens entscheidend für reine Schönheit als Gesamtkunstwerk ist, so wäre es doch falsch, die Schönheit der Einzelpflanze zu unterschätzen $« .{ }^{23}$ Während andere Planer die wiederentdeckte Staudenvielfalt im

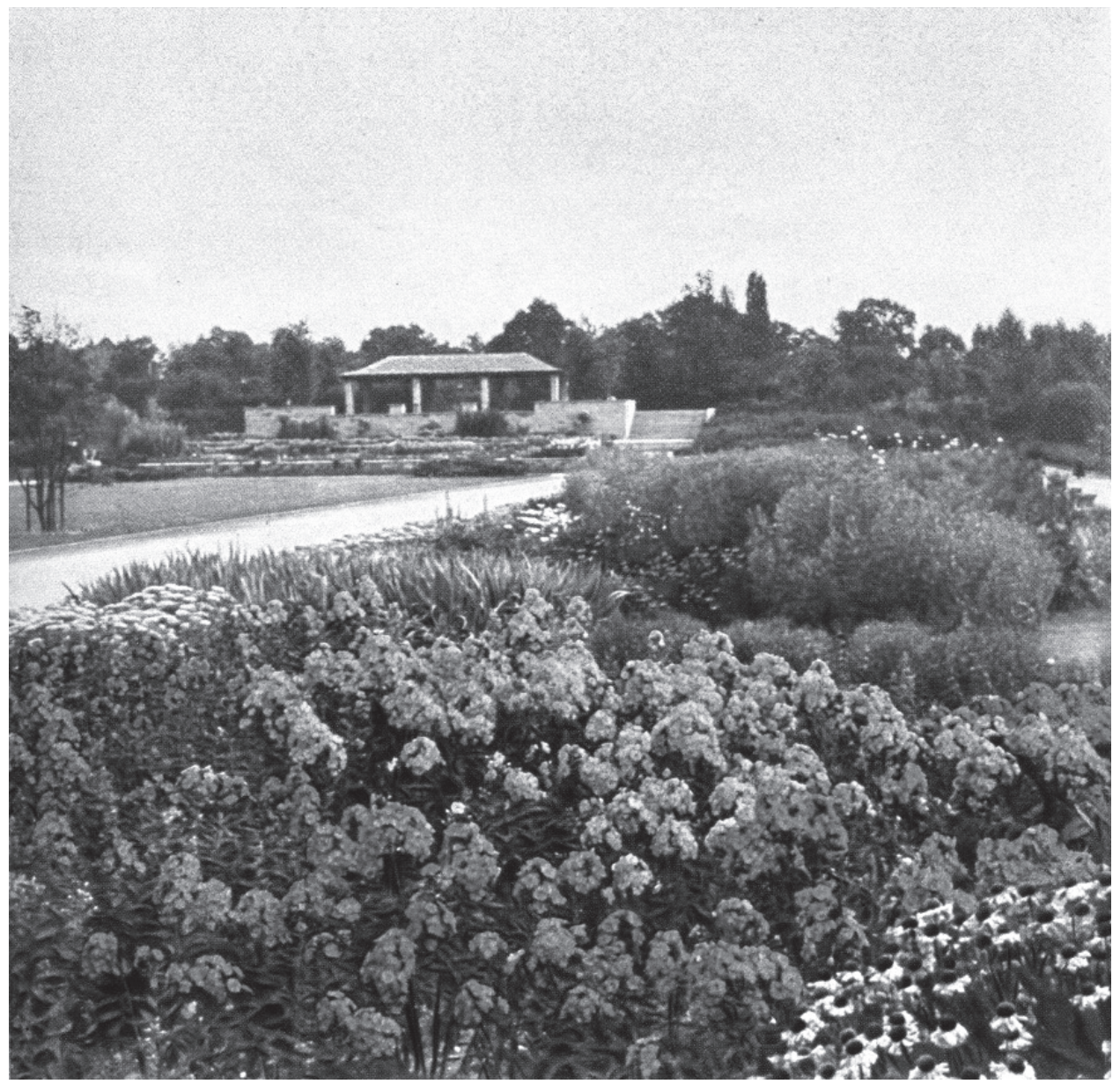

4 | Staudenrabatte von Gustav Allinger im Richard-Wagner-Hain in Leipzig, Planung um 1934, Fotografie 1941 (Die Gartenkunst, 1941, S. 51). 
neuen Wohngarten einsetzten, arbeitete Allinger wieder in monumentalem Umfang. Doch im Gegensatz zu üblichen Beetmaßen vergrößerte Allinger im Richard-WagnerHain seine Pflanzung auf 12 Meter Breite und 225 Meter Länge. Blockweise und rhythmische Staudenpflanzungen führten nicht nur zu einer künstlerisch-grafischen Ordnung, sondern auch zu einer Intensivierung des erlebten Farbeindrucks und einer überzeugenden Fernwirkung. Um Blühpausen der Stauden zu überbrücken, füllte er wie in der Jugend bei Encke erlernt - die große Staudenrabatte mit Sommerblumen auf. Man muss sich Allingers Rabatten und Beete in für heutige Verhältnisse ungewöhnlich bunter und leuchtender Farbkraft vorstellen. Bei der Gartengestaltung dürfte die Farbgebung der Materialien und Gartenausstattungen auf keinen Fall mit den kräftigen Blütenfarben konkurrieren. ${ }^{24}$ Menschen durch Pflanzungen glücklich zu machen, ist aber zu keiner Zeit verkehrt, doch bleibt ein unguter Beigeschmack, wenn sich Allinger hier ausdrücklich auf ein »Kraft durch Freude «-Konzept beruft. ${ }^{25}$

\section{Fazit}

Zusammenfassend bleibt festzustellen, dass Allinger in Gesamtplanung und Pflanzenverwendung gerne auf große Gesten setzte. Während er in den späten 1920er Jahren mit den plakativen, farbintensiven teppichbeetartigen Pflanzungen offenbar genau den Zeitgeist der Besucher getroffen hatte, findet man in der Fachliteratur zu Allingers über-

\section{Anhang}

Bepflanzung der großen Staudenrabatte im Richard-Wagner-Hain in Leipzig (Abb. 4): ${ }^{27}$

Blumenzwiebeln: Frühjahrsflor mit einfachen frühen Tulpen in Sorten (i.S.), einfachen späten Tulpen i.S. und Darwin-Tulpen i.S. proportional großen Staudenachsen und Sondergärten der 1930er Jahre kaum Kommentare von Kollegen oder zeitgenössischen Nutzern. In den 1920er Jahren war Allinger noch ganz von den sozialreformerischen Leitbildern der vorausgegangenen Epoche der Reformgartenzeit erfüllt und hatte fast spielerisch-experimentell gepflanzt. In den 1930er Jahren wurden der Anlass und die Stimmung der Pflanzung deutlich ernster. Allinger pflanzte in den 30er Jahren bewusster und mit mehr Erfahrung. Doch wird immer mehr eine Monumentalität in den Vordergrund gestellt, kommt keine wirkliche Hausgartenatmosphäre auf, die in den Reformgartenparkanlagen fehlendes privates Grün ersetzten konnte. Hier geht Allinger deutlich andere Wege als sein Vorbild Fritz Encke, der sich immer an menschlichen Maßen und Ansprüchen orientiert hatte. Allinger beschreibt in seinem unveröffentlichten Manuskript »Gartengestaltung und Gartenkunst in Vergangenheit und Gegenwart « den Hamburger Stadtpark als »sozialen Park unserer Zeit«, ohne »repräsentative Bedürfnisse nach Prunk und nach Luxus«, entstanden »aus der Not, die für uns Großstadtmenschen erwächst durch das Verschwinden eines lebendigen Verhältnisses zur Natur «. ${ }^{26}$ Es bleibt fraglich, ob Allinger seine eigenen Bewertungsmaßstäbe bei seinen drei großen Parkanlagen der 1930er Jahre erfüllt hat. Sie sind nicht luxuriös, aber in hohem Maße repräsentativ. Die Pflanzungen setzten die Besucher mit Sicherheit in erfreutes Erstaunen, doch nicht in ein lebendiges Verhältnis zur Natur.

Höhere Stauden: Frühsommer-Auftakt mit blauen und weißen Stauden-Lupinen (Lupinus polyphyllus), weißer Garten-Margerite (Leucanthemum x superbum i.S.) und orangerotem Orientalischen Mohn (Papaver orientale). Im Hochsommer und Spätsommer folgen blauer Rittersporn (Delphinium 


\section{Die Pflanzenverwendung von Gustav Allinger}

i.S.), blauviolette Berg-Astern (Aster amellus i.S.), violetter Großblütiger Ziest (Stachys grandiflora), intensiv gelbe und orangefarbene Sonnenbraut-Sorten (Helenium hoopesii, Helenium hybr. 'Riverton Gem, 'Crimson Beauty', 'Wyndley'), Bunte Margerite (Tanacetum coccineum i.S.), hohe Raublatt-Aster (Aster novae-angliae), Feinstrahlaster (Erigeron), Staudenphlox (Phlox paniculata in roten, rosafarbenen und lachsfarbenen Sorten, u.a. 'Leuchtfeuer' und 'Carmen'), niedriger Sonnenhut (Rudbeckia fulgida var. speciosa) und hoher Sonnenhut (Rudbeckia nitida).

Niedrige und Polsterstauden: Grasnelke (Armeria maritima), Alpen-Astern (Aster alpinus), Blaukissen (Aubrieta hybr. i.S.), Filziges Hornkraut (Cerastium tomento- sum), Quirlblättriges Mädchenauge (Coreopsis verticillata), Lerchensporn (Corydalis cheirantifolia), Herzblättriger Gämswurz (Doronicum columnae), Vielfarbige Wolfsmilch (Euphorbia polychroma), niedrige Schwertlilie (Iris pumila), Missouri-Nachtkerze (Oenothera macrocarpa), Kelch-Phlox (Phlox amoena), Polster-Phlox (Phlox subulata), Fetthenne (Sedum spectabile), WollZiest (Stachys byzantina) und Hornveilchen (Viola cornuta i.S.).

Sommerblumen: Löwenmäulchen (Antirrhinum majus i.S.), einjährige Astern, Roter Fingerhut (Digitalis purpurea), Studentenblumen (Tagetes erecta i.S., Tagetes patula i.S.), Eisenkraut (Verbena hybr.) und Zinnien (Zinnia elegans i.S.).
1 Allinger, Gustav: Die Schmuckanlage in der Dresdener Gartenbau-Ausstellung 1926, in: Gartenschönheit, Heft 4/1927, S. 85.

2 Haenel, Erich: Gartenschau in Dresden, in: Die Kunst. Monatshefte für freie und angewandte Kunst, Band 56 1926, S. 57f, hier: S. 60.

3 Rottenberger, Fritz: Die Eröffnung der Jubiläumsgartenbauausstellung in Dresden am 23. April 1926, in: Gartenzeitung der Oesterreichischen Gartenbaugesellschaft in Wien, 1926, S. 53f, hier: S. 53.

4 Schneider, Camillo: Dresdens Gartenschau. Ein Rückblick, in: Gartenschönheit, Heft 11/1926, S. 290-293, hier: S. 290.

5 Ebd., S. 290.

6 Redaktion Möller's Deutsche Gärtner-Zeitung: Das Teppichbeet. Was halten Sie vom Teppichbeet und wie denken Sie über seine künstlerische Wiedergeburt, in: Möller's Deutsche Gärtner-Zeitung 1912, S. $540 \mathrm{f}$.

7 Kammeyer, Hans Felix: Die Blume als Massenwirkung in unseren Gärten, in: Möller's Deutsche Gärtner-Zeitung 1918, S. 90-99, hier: S. 90.

8 Migge, Leberecht: Das Teppichbeet. Was halten Sie vom Teppichbeet und wie denken Sie über seine künstlerische Wiedergeburt, in: Möller's Deutsche Gärtner-Zeitung 1912, S. 540f, hier: S. 544; Migge, Leberecht: Die Grundgedanken der Leipziger Ausstellungsgärten, in: Möller's Deutsche Gärtner-Zeitung 1913, S. 603-606, hier: S. 606.

9 Koeppner, Robert: Kann die Gartenkunst expressionistisch sein? In: Gartenflora 1931, S. 86-90, hier: S. 89.

10 Foerster, Karl: Blumenzwiebelbuch, Berlin 1939, S. 37.
11 Allinger, Gustav: Der Hausgarten von heute, in: Gartenschönheit 1936, S. 200-203, hier: S. 200.

12 Ebd. S. 200.

13 Adams, Werner; Joachim Bauer (Hg.): Vom Botanischen Garten zum Großstadtgrün. 200 Jahre Kölner Grün, Köln 2001, S. 124.

14 Ebd. S. 23.

15 Allinger, Gustav: Gartengestaltung und Gartenkunst in Vergangenheit und Gegenwart, Band 1/ Sammlung an Manuskripten, Berlin/Erfurt o.D. (nach 1945), unveröffentlicht, S. 19.

16 Allinger, Gustav: Der deutsche Garten. Sein Wesen und seine Schönheit in alter und neuer Zeit, München 1950, S. 232.

17 Ebd. S. 43.

18 Allinger, Gustav: Vom Wesen der Form in der Garten- und Landschaftsgestaltung, in: Die Gartenkunst 1941, S. 37-53, hier: S. 50.

19 Ebd. S. 53.

20 Allinger, Gustav: Gartengestaltung und Gartenkunst in Vergangenheit und Gegenwart, Band 2/ Abbildungen, Berlin/Erfurt o.D. (nach 1945), unveröffentlicht, S. 13.

21 Allinger 1941, S. 43.

22 Ebd. S. 43.

23 Allinger 1950 S. 228.

24 Ebd. S. 119.

25 Allinger 1941, S. 53.

26 Allinger o.D. Bd. 1, S. 19.

27 Allinger 1941, S. 52, ergänzt durch Informationen aus Pflanzplänen von Gustav Allinger, 1934; Pflanzennamen nach aktueller Nomenklatur (Erhardt, Götz, Bödeker, Seybold, 2008) überarbeitet 